PROCEEDINGS OF THE

AMERICAN MATHEMATICAL SOCIETY

Volume 132, Number 8, Pages 2241-2246

S 0002-9939(04)07409-X

Article electronically published on March 25, 2004

\title{
THE HILTON-HECKMANN ARGUMENT FOR THE ANTI-COMMUTATIVITY OF CUP PRODUCTS
}

\author{
MARIANO SUAREZ-ALVAREZ
}

(Communicated by Martin Lorenz)

\begin{abstract}
We present a simple extension of the classical Hilton-Eckmann argument which proves that the endomorphism monoid of the unit object in a monoidal category is commutative. It allows us to recover in a uniform way well-known results on the graded-commutativity of cup products defined on the cohomology theories attached to various algebraic structures, as well as some more recent results.
\end{abstract}

\section{INTRODUCTION}

In their paper [1] on group objects in general categories, B. Eckmann and P. J. Hilton show that two monoid structures on a set which are such that one of them is a homomorphism for the other actually coincide and are commutative; this is stated - for groups - as Theorem 5.4.2 in that paper.

Interestingly, this situation does occur in nature: if $X$ and $Y$ are respectively a co- $H$-space and an $H$-space, then the set of based homotopy classes of maps [ $X, Y]$ is a monoid in two ways, and naturality implies that each of the corresponding products on $[X, Y]$ is a homomorphism for the other. The result just quoted implies then that these two structures are equal, and that $[X, Y]$ is commutative. Instances of this are the well-known facts that both the fundamental group $\pi_{1}(G, e)$ of a topological group $G$ and the higher homotopy groups $\pi_{p}\left(X, x_{0}\right)$ for $p>1$ of any space $X$ are abelian; see section 5.3 in [1].

Another situation in which the same argument applies is the following. Let $\mathrm{C}$ be a monoidal category with unit object $e \in \operatorname{objC}$. The set end $\mathrm{C}(e)$ of endomorphisms of $e$ in $\mathrm{C}$ is of course an associative monoid with respect to composition. It turns out that it is always commutative- $c f$. [6], Proposition XI.2.4. Indeed, on $\operatorname{end}_{\mathrm{c}}(e)$ we can define a convolution product and verify that it is a homomorphism with respect to composition. It follows that composition and convolution in $\operatorname{end}_{\mathrm{C}}(e)$ are equal and commutative. We refer to Ch. Kassel's book [6] for details.

The purpose of this note is to present a simple extension of the argument of Eckmann and Hilton which can be used to show that products defined on various cohomology theories are commutative; it can be seen as a "derived" version of 6], Proposition XI.2.4. It applies to the cohomology of groups, to the Hochschild

Received by the editors October 23, 2002 and, in revised form, May 10, 2003.

2000 Mathematics Subject Classification. Primary 18E30, 18G99; Secondary 16E40.

This work was supported by a grant from UBACYT X062, the international cooperation project SECYT-ECOS A98E05, and a CoNICET scholarship. 
cohomology of associative algebras, to the Cartier cohomology of coalgebras, and to other somewhat more exotic theories, such as the cohomology theory introduced by M. Gerstenhaber and S. D. Schack in [5] for Hopf bimodules over a Hopf algebra.

In the next section we recall the definition of a monoidal category so as to fix the notation, we extend it in a natural way in order to adapt it to a "derived" context, and we state and prove our theorem. In section 2 we indicate how our result allows us to establish easily the graded commutativity of the product on the usual cohomology theories.

\section{DEFinitions AND THE THEOREM}

1.1. In what follows all categories and functors are implicitly assumed to be additive.

Definition 1.2. A monoidal category is a 6 -tuple $(\mathrm{C}, \otimes, e, a, l, r)$ in which $\mathrm{C}$ is a category, $? \otimes ?: \mathrm{C} \times \mathrm{C} \rightarrow \mathrm{C}$ a bifunctor, $e \in$ obj $\mathrm{C}$ an object, $a:(? \otimes ?) \otimes ? \rightarrow ? \otimes(? \otimes ?)$ an isomorphism of functors $\mathrm{C} \times \mathrm{C} \times \mathrm{C} \rightarrow \mathrm{C}$, and in which $l: e \otimes ? \rightarrow ?$ and $r: ? \otimes e \rightarrow$ ? are isomorphisms of functors $\mathrm{C} \rightarrow \mathrm{C}$, which are such that for each choice of objects $x, y, z$ and $w \in \operatorname{obj} \mathrm{C}$, the following diagrams commute:

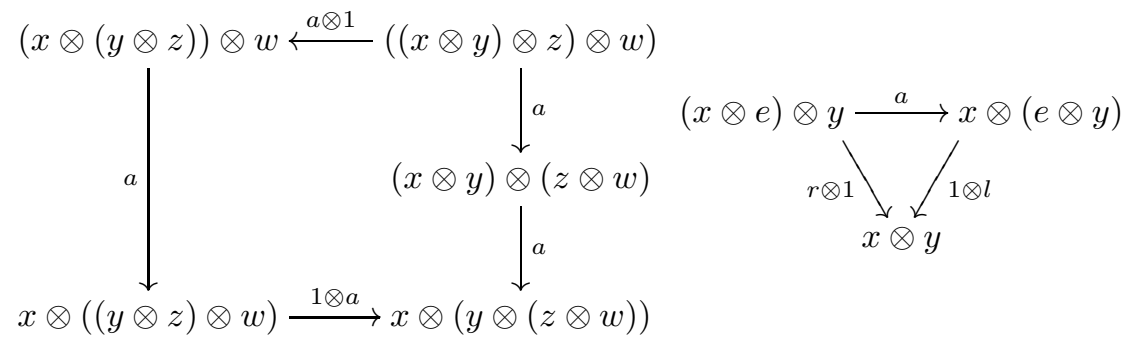

1.3. It follows easily from the definition that $l=r: e \otimes e \rightarrow e$; see for example [6], Lemma XI.2.3. The proof of this fact involves the associativity constraint $a$, and it is interesting to notice that this is the only rôle played by $a$ in what follows.

Definition 1.4. A suspended monoidal category is a 9-tuple $(\mathrm{C}, \otimes, e, a, l, r, T, \lambda, \rho)$ such that $(\mathrm{C}, \otimes, e, a, r, l)$ is a monoidal category, $T: \mathrm{C} \rightarrow \mathrm{C}$ is an automorphism, and $\lambda: ? \otimes T ? \rightarrow T(? \otimes ?)$ and $\rho: T ? \otimes ? \rightarrow T(? \otimes ?)$ are isomorphisms of functors $\mathrm{C} \times \mathrm{C} \rightarrow \mathrm{C}$ such that for each pair of objects $x$ and $y \in \operatorname{objC}$, the following two diagrams commute:

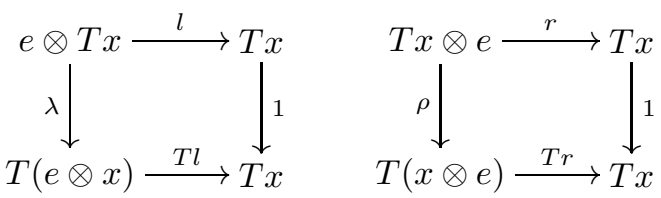

while the following diagram anti-commutes:

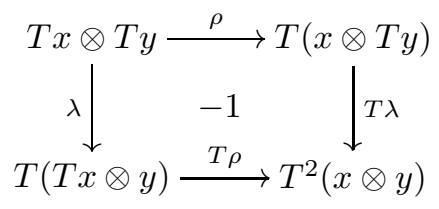


1.5. Given a suspended monoidal category as in 1.4, we put $\lambda_{0}=\rho_{0}=1: x \otimes y \rightarrow$ $x \otimes y$, and for each $p>0$,

$$
\begin{aligned}
\lambda_{p} & =T^{p-1} \lambda \circ T^{p-2} \lambda \circ \cdots \circ \lambda: x \otimes T^{p} y \rightarrow T^{p}(x \otimes y), \\
\rho_{p} & =T^{p-1} \rho \circ T^{p-2} \rho \circ \cdots \circ \rho: T^{p} x \otimes y \rightarrow T^{p}(x \otimes y),
\end{aligned}
$$

and

$$
\begin{aligned}
& \lambda_{-p}=T^{-p} \lambda_{p}^{-1}: x \otimes T^{-p} y \rightarrow T^{p}(x \otimes y), \\
& \rho_{-p}=T^{-p} \rho_{p}^{-1}: T^{-p} x \otimes y \rightarrow T^{p}(x \otimes y) .
\end{aligned}
$$

Observe that these last two equations do make sense: for example, since $\lambda_{p}$ : $x \otimes T^{p} T^{-p} y \rightarrow T^{p}\left(x \otimes T^{-p} y\right)$, we have $\lambda_{p}^{-1}: T^{p}\left(x \otimes T^{-p} y\right) \rightarrow x \otimes T^{p} T^{-p} y$, so $T^{-p} \lambda_{p}^{-1}: x \otimes T^{-p} y \rightarrow T^{-p}(x \otimes y)$.

1.6. We obtain in this way isomorphisms of functors $\lambda_{p}: ? \otimes T^{p} ? \rightarrow T^{p}(? \otimes ?)$ and $\rho_{p}: T^{p} ? \otimes ? \rightarrow T^{p}(? \otimes ?)$ for all $p \in \mathbb{Z}$ such that we have

$$
\begin{gathered}
l=T^{p} l \circ \lambda_{p}: e \otimes T^{p} ? \rightarrow T^{p} ?, \\
r=T^{p} r \circ \rho_{p}: T^{p} ? \otimes e \rightarrow T^{p} ?, \\
T^{p} \lambda_{q} \circ \rho_{p}=(-1)^{p q} T^{q} \rho_{p} \circ \lambda_{q}: T^{p} ? \otimes T^{q} ? \rightarrow T^{p+q}(? \otimes ?),
\end{gathered}
$$

for all $p, q \in \mathbb{Z}$.

Theorem 1.7. Let $(\mathrm{C}, \otimes, e, a, l, r, T, \lambda, \rho)$ be a suspended monoidal category, and put

$$
\operatorname{end}_{C}^{T}(e)=\bigoplus_{p \in \mathbb{Z}} \operatorname{hom}_{C}\left(e, T^{p} e\right)
$$

If $f: e \rightarrow T^{p} e$ and $g: e \rightarrow T^{q} e$, define $f \cdot g=T^{q} f \circ g: e \rightarrow T^{p+q} e$. Then $\left(\operatorname{end}_{C}^{T}(e), \cdot\right)$ is a commutative graded ring.

Proof. The facts that the operation - is associative, that it admits $1 \in \operatorname{hom}_{C}(e, e)$ as a unit element, and that it is distributive with respect to addition follow immediately from the corresponding facts about the composition of morphisms in C. We need only prove then that it is commutative.

If $f: e \rightarrow T^{p} e$ and $g: e \rightarrow T^{q} e$, we let $f \star g: e \rightarrow T^{p+q} e$ be the composition

$$
e \stackrel{r^{-1}}{\longrightarrow} e \otimes e \stackrel{f \otimes g}{\longrightarrow} T^{p} e \otimes T^{q} e \stackrel{\rho_{p}}{\longrightarrow} T^{p}\left(e \otimes T^{q} e\right) \stackrel{T^{p} \lambda_{q}}{\longrightarrow} T^{p+q}(e \otimes e) \stackrel{T^{p+q} l}{\longrightarrow} T^{p+q} e .
$$

Each bounded face in the following diagram commutes:

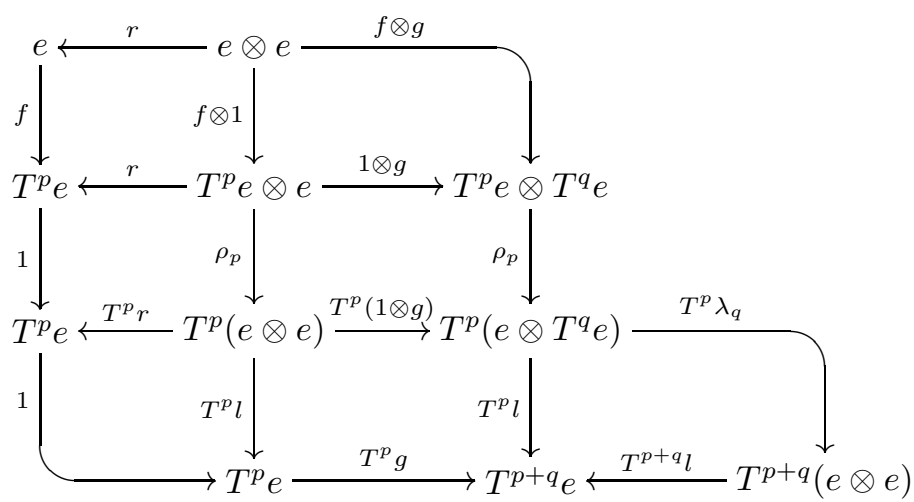


So the outer one does, too, and we see that $f \star g=g \cdot f$. On the other hand, from the $(-1)^{p q}$-commutativity of

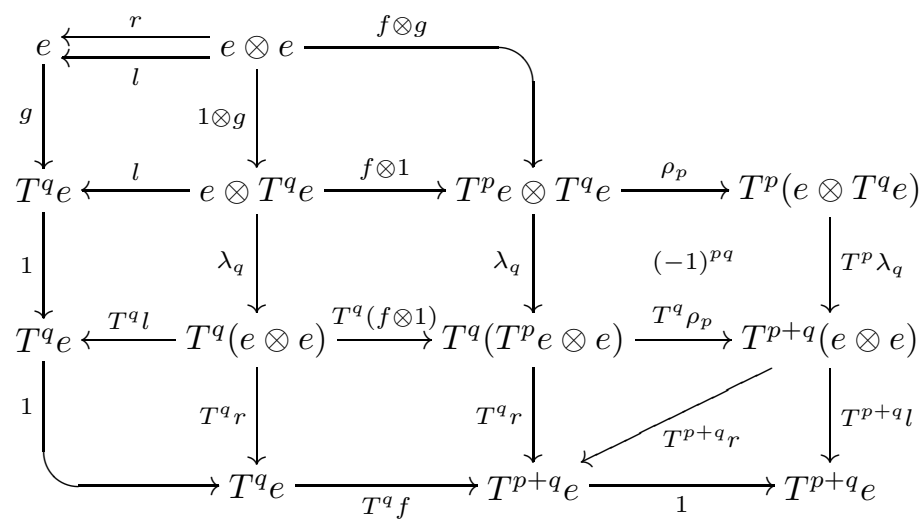

we see that we also have $f \star g=(-1)^{p q} f \cdot g$. It follows from this, of course, that $\cdot$ is a graded commutative operation.

\section{Applications}

2.1. Suppose $(\mathrm{C}, \otimes, e, a, l, r)$ is a monoidal category such that the underlying category $\mathrm{C}$ is an exact category, and such that the tensor product is an exact functor. Then $\otimes$ can be extended naturally to the bounded derived category $\mathrm{D}^{b}(\mathrm{C})$, and it is not difficult to see that $\mathrm{D}^{b}(\mathrm{C})$ becomes in this way a suspended monoidal category, whose unit object is simply $e$ considered as an object of $D^{b}(C)$ in the usual way, and whose suspension functor is the translation; we refer to [2] for general information on derived categories and functors.

Our theorem now implies that $\operatorname{Ext}_{\mathrm{C}}^{\bullet}(e, e)=\bigoplus_{p \in \mathbb{Z}} \operatorname{hom}_{\mathrm{D}^{b}(\mathrm{C})}\left(e, T^{p} e\right)$ is commutative for the composition product considered in 1.7, which of course is simply the Yoneda product.

2.2. This applies in particular to the abelian monoidal category $\mathrm{C}={ }_{H}$ Mod of left $H$-modules over a Hopf algebra $H$ defined over a field $k$, with tensor product induced by the tensor product $\otimes_{k}$ of vector spaces endowed with diagonal action. Since the unit object is $k$ with trivial action, we conclude that $\operatorname{Ext}_{H}^{\bullet}(k, k)$ is commutative for the Yoneda product.

Dually, the Yoneda algebra $\operatorname{Ext}_{H}^{\bullet}(k, k)$ of self-extensions of $k$ in the monoidal category ${ }^{H}$ Mod of left $H$-comodules is commutative.

2.3. A well-known instance of this situation arises when $H=k G$ is the group algebra of a group $G$ over a commutative base ring $k$. We recover in this way the fact that $H^{\bullet}(G)=\operatorname{Ext}_{k G}^{\bullet}(k, k)$, the group cohomology of $G$ over $k$, is a commutative ring for the Yoneda product. Since this coincides with the cup product on $H^{\bullet}(G)$, we recover the fact that the cup product is commutative.

2.4. We remark that all that is really needed in order to be able to conclude in this way that $\operatorname{Ext}_{H}^{\bullet}(k, k)$ is commutative is a monoidal structure on ${ }_{H}$ Mod for which $k$ is the unit object, and this monoidal structure need not arise from a bialgebra structure on $H$. 
Thus, if $H$ is only a quasi-bialgebra in the sense of Drinfel'd - see for example [6], Chapter XV — we reach the same conclusion.

2.5. The theorem can also be applied in situations in which the tensor product $\otimes$ in the initial datum is not exact but can be extended by derivation to the derived category.

For example, if $A$ is a possibly non-commutative $k$-algebra, then the category $A^{e}$ Mod of $A$-bimodules can be endowed with the structure of a monoidal category with product given by the usual tensor product $\otimes_{A}$ of $A$-modules, which is in general not an exact functor. It is right exact, though, and so it does admit the left derived functor $\otimes_{A}^{L}$ as an extension on the derived category $\mathrm{D}^{-}\left(A^{e} \mathrm{Mod}\right)$ of bounded above complexes, which in this way naturally becomes a suspended monoidal categoryindeed, Definition 1.4 is designed to capture precisely this situation. The unit object is clearly $A \in \operatorname{obj} D^{-}\left(A^{e}\right.$ Mod).

Our theorem implies then that the Hochschild cohomology of $A, H H^{\bullet}(A)=$ $\operatorname{Ext}_{A^{e}}(A, A)$, is commutative for the Yoneda product. Now, since the Yoneda product on $H H^{\bullet}(A)$ is the same as the cup product defined by M. Gerstenhaber in [3], 4, we recover the fact that this last product is commutative.

We note that this argument can be dualized to obtain a proof that the Yoneda product on the Cartier cohomology $H H^{\bullet}(C)$ of a coalgebra $C$, or equivalently, the algebra $\operatorname{Ext}_{C^{e}}(C, C)$ of self-extensions of $C$ in the category ${ }^{C} \operatorname{Mod}^{C}$ of $C$ bicomodules, is commutative.

2.6. For our final example, let $H$ be a Hopf algebra over a field $k$, and let us consider the abelian category $\mathcal{M}={ }_{H}^{H} \operatorname{Mod}_{H}^{H}$ of Hopf $H$-bimodules. It can be made into a monoidal category with product given by the tensor product $\otimes_{H}$ of $H$-bimodules in such a way that the forgetful functor $\mathcal{M} \rightarrow{ }_{H} \operatorname{Mod}_{H}$ is monoidal. If $M, N \in \mathcal{M}$, then $M \otimes_{H} N$ has its left module structure induced by that on $M$, its right module structure induced by that on $N$, and codiagonal left and right comodule structures. We refer to P. Schauenburg's paper [7] for details.

The unit object is $H$, and the product is exact because one-sided Hopf bimodules are free. We are thus in the situation of 2.1, and we conclude that the Yoneda product on the extension algebra $\operatorname{Ext}_{\mathcal{M}}^{\bullet}(H, H)$ is commutative.

This result has been obtained in a different way by R. Taillefer in [8, section 4 . Since she has shown in 9] that the Yoneda algebra $\operatorname{Ext}_{\mathcal{M}}^{\bullet}(H, H)$ is isomorphic to the cohomology groups $H_{G S}^{\bullet}(H, H)$ introduced by Gerstenhaber and Schack when these are endowed with a certain (rather complex) cup product, we see that this last product is commutative.

We note in passing that the category $\mathcal{M}$ can be endowed with another monoidal structure, with product induced by the cotensor product $\square^{H}$ of $H$-bicomodules, in such a way that now the forgetful functor $\mathcal{M} \rightarrow{ }^{H} \operatorname{Mod}^{H}$ is monoidal. We do not really obtain anything new, on the one hand because the identity functor $\mathcal{M} \rightarrow \mathcal{M}$ is (in a nontrivial way) a monoidal equivalence $\left(\mathcal{M}, \otimes_{H}\right) \cong\left(\mathcal{M}, \square^{H}\right)$, as shown in [7, Corollary 6.1, and because the conclusion in Theorem 1.7] is actually independent of the particular monoidal structure under consideration. However, in view of the equality $f \star g=g \cdot f$ obtained in the course of the proof of the theorem, this shows that the cup product on $\operatorname{Ext}_{\mathcal{M}}^{\bullet}(H, H)$ can be computed using either the $\star$-product defined in terms of $\otimes_{H}$ or the one defined in terms of $\square^{H}$, and this observation might lead to simplifications when doing actual computation. 


\section{REFERENCES}

1. B. Eckmann and P. J. Hilton, Group-like structures in general categories. I. Multiplications and comultiplications, Math. Ann., 145, 1961/1962, 227-255. MR 25:108

2. Sergei I. Gelfand and Yuri I. Manin, Methods of homological algebra, Springer Monographs in Mathematics, 2, Springer-Verlag, Berlin, 2003, xx+372 pp. MR 2003m:18001

3. Murray Gerstenhaber, The cohomology structure of an associative ring, Ann. of Math. (2), 78, 1963, 267-288. MR 28:5102

4. Murray Gerstenhaber On the deformation of rings and algebras, Ann. of Math. (2), 79, 1964, 59-103. MR 30:2034

5. Murray Gerstenhaber and Samuel D. Schack, Bialgebra cohomology, deformations, and quantum groups, Proc. Nat. Acad. Sci. U.S.A., 87, No. 1, 1990, 478-481. MR 90j:16062

6. Christian Kassel, Quantum groups, Graduate Texts in Mathematics, 155, Springer-Verlag, New York, 1995, xii+531 pp. MR 96e:17041

7. Peter Schauenburg, Hopf modules and Yetter-Drinfel'd modules, J. Algebra, 169, No. 3, 1994, 874-890, MR 95j:16047

8. Rachel Taillefer, Cohomology theories of Hopf bimodules and cup-product, C. R. Acad. Sci. Paris Sér. I Math., 332, No. 3, 2001, 189-194. MR 2001m:16066

9. Rachel Taillefer, Injective Hopf bimodules, cohomologies of infinite dimensional Hopf algebras and graded-commutativity of the Yoneda product.

Departamento de Matemática, Facultad de Ciencias Exactas y Naturales, Universidad de Buenos Aires, Ciudad Universitaria, Pabellón I, Buenos Aires (1428), Argentina

E-mail address: mariano@dm.uba.ar

URL: http://mate.dm.uba.ar/ aldoc9 